\title{
Primary Care Providers: Discuss COVID-19-Related Goals of Care with Your Vulnerable Patients Now
}

\author{
Eric Kutscher, $M D^{1,2}$ and Mat Kladney, $M D^{1,2}$ \\ 'Internal Medicine, NYU Langone Health, New York, NY, USA; ${ }^{2}$ Internal Medicine, Bellevue Hospital Center, New York, NY, USA.
}

J Gen Intern Med 35(7):2195-6

DOI: $10.1007 / \mathrm{s} 11606-020-05862-7$

(c) Society of General Internal Medicine 2020

$\mathrm{S}$ he panted for breath while trying to tell me about her elderly mother's symptoms over the COVID hotline. The caller, so concerned about her mother's health, had failed to notice she herself had a fever and cough for the past few days. Her mother's symptoms sounded stable, I assured her, but her own condition was dangerous. I pleaded for her to go to the emergency room for evaluation for breathing support. As she agreed and disconnected, I went on to the next caller.

Her case was far from unique. I repeatedly heard myself explaining that with no cure for COVID-19, the most important thing to monitor was breathing: if at any point someone felt winded, unable to carry on a conversation, or were gasping for air, they should present to the emergency department immediately to be evaluated for breathing support.

After saying this dozens of times, I realized I never prepared the callers for what to expect in the emergency department. I never explained that by "breathing support," I meant consideration for intubation and mechanical ventilation. Many of the tools we have traditionally used to "support breathing" have been limited in supply, and even when available, BIPAP, CPAP, and HFNC are all considered to be highly aerosolizing procedures to be limited in use as they put others at risk for contracting COVID. Without these options, we are often left with two extremes: nasal cannula and nonrebreather facemasks versus intubation.

It occurred to me: if I am not preparing these callers for intubation, who is? Is intubation something these individuals would even want? And would having a conversation with someone they trust before getting sick be better than when they are sick, or even worse, never at all?

Conversations about intubation are usually had infrequently and too late. While intubation has its clear benefits of prolonging life, especially in scenarios with reversible illness, it has significant risks that we rarely talk about with our

Received March 31, 2020

Accepted April 13, 2020

Published online May 6, 2020 patients. It is difficult to discuss the possible long-term consequences of intubation because frankly we cannot predict if these complications will occur for each patient. We do not know who will require increased pressures or $\mathrm{FiO} 2$, resulting in sustained lung injuries. We do not know who will breathe asynchronously with the ventilator, thus requiring increasing doses of sedatives. We do not know who will simply die from their underlying illness, despite mechanical ventilation. We do not know who will survive to extubation, only to ultimately succumb to pneumonia or urosepsis while undergoing rehabilitation. All of these unknowns often result in doctors uncomfortable in talking about risks and benefits of intubation with their patients until it is clear that the patient has progressed to a point of needing respiratory support. And there is, inherently, never a right moment to have this conversation.

Uniquely, many of the unknowns about intubation are answered with data on COVID-19 from other countries. Patients often require a prolonged intubation of 10-14 days, and patients who are older, have cardiovascular disease (including hypertension), diabetes, or malignancy have worse outcomes. ${ }^{1,2}$ Evidence from the China CDC shows that in Wuhan, $49 \%$ of critically ill patients died. ${ }^{2}$ Looking specifically at those intubated, data published from China shows small numbers of intubations with alarming mortality rates. Yang et al. show a mortality rate of 19 out of 21 intubated patients. ${ }^{3}$ Zhou et al. show 31 of 32 intubated patients dying prior to discharge. ${ }^{1}$ In the largest study by Guan et al., all 25 intubated patients died. ${ }^{4}$ For many in the United States, patients who have died while intubated have been alone and without visitors. ${ }^{5}$ For those that do survive, the risk of cachexia and a prolonged recovery is almost inevitable.

Volunteering as the anonymous doctor on a COVID hotline, it was clearly not my role to discuss with these callers whether or not intubation is something they would want. It was instead my role to triage concerns and get people complete medical evaluation if needed. Yet, I felt a dissonance: a conversation I felt like I ought to have but could not.

In the emergency department, it is not the job of the ED physician to explain intubation to a decompensating patient needing emergent intervention. With thousands of expected cases of COVID causing respiratory distress at the same time here in New York City, it is even more unrealistic for us to expect our emergency medicine colleagues to have long nuanced goals of care conversations in the acute setting. This is 
exacerbated by new visitation policies at most NYC hospitals barring all visitors for adult patients.

We as primary care doctors have the privilege of knowing our patients the best. We identify as their doctor and their advocate. Through our repeated encounters, our patients learn to trust us, and we learn to trust them. Thus, in this time of medical crisis, we must step up to help patients better understand this pandemic. We must reach out to our most vulnerable patients and take advantage of our deep relationships to have difficult conversations. We must ask our patients about their concerns about COVID-19, and share information about how to avoid the virus. We must also ask our patients about what medical interventions they would want if they were to contract COVID-19 and require respiratory support. We must be clear and transparent in our thoughts and recommendations, tailoring them to each individual, and communicating them with our best intentions to help patients find a path that is right for them. Given the data we know about COVID-19 and the risks and benefits of intubation, we must use our best medical judgment to help patients understand realistic outcomes and make informed decisions.

For many of our conversations, we may not reach a conclusion as to how to best address respiratory support in this pandemic. But by at least opening the conversation and discussing the options, our patients can be active participants in their care. For those who ultimately decide that intubation is outside of their goals, we must help document these wishes through proper legal forums to make sure their desires are respected.

The role of the primary care doctor is to partner with our patients to help them find their voice in the medical system. With COVID-19, this means having hard conversations with our most vulnerable patients. It means helping many of our beloved patients understand that "do not intubate" is most likely the best choice for them.

Corresponding Author: Eric Kutscher, MD; Internal Medicine, Bellevue Hospital Center, New York, NY, USA (e-mail: Eric. Kutscher@nyulangone.org).

\section{Compliance with ethical standards:}

Conflict of interest: Authors have no conflicts of interest to declare.

\section{REFERENCES}

1. Zhou F, Yu T, Du R, et al. Clinical course and risk factors for mortality of adult inpatients with COVID-19 in Wuhan, China: a retrospective cohort study [published online ahead of print, 2020 Mar 11] [published correction appears in Lancet. 2020 Mar 12;:]. Lancet. 2020;S0140-6736(20)30566-3. doi:https://doi.org/10.1016/S0140-6736(20)30566-3

2. The Novel Coronavirus Pneumonia Emergency Response Epidemiology Team. The Epidemiological Characteristics of an Outbreak of 2019 Novel Coronavirus Diseases (COVID-19) - China, 2020[J]. China CDC Weekly, 2020, 2(8): 113-122.

3. Yang $\mathbf{X}, \mathbf{Y u} \mathbf{Y}, \mathbf{X u} \mathbf{J}$, et al. Clinical course and outcomes of critically ill patients with SARS-CoV-2 pneumonia in Wuhan, China: a single-centered, retrospective, observational study [published online ahead of print, 2020 Feb 24] [published correction appears in Lancet Respir Med. 2020 Feb 28;:]. Lancet Respir Med. 2020;S2213-2600(20)30079-5. doi:https:// doi.org/10.1016/S22 13-2600(20)30079-5

4. Guan WJ, Ni ZY, Hu Y, et al. Clinical Characteristics of Coronavirus Disease 2019 in China [published online ahead of print, $2020 \mathrm{Feb} 28$ ]. $N$ Engl J Med. 2020; https://doi.org/10.1056/NEJMoa2002032.

5. Lamas D. Opinion: I'm on the Front Lines. I Have No Plan for This. The New York Times. https://www.nytimes.com/2020/03/24/opinion/coronavirus-hospital-visits.html. Published 2020. Accessed March 26, 2020.

Publisher's Note: Springer Nature remains neutral with regard to jurisdictional claims in published maps and institutional affiliations. 